Trauma Berufskrankh $2014 \cdot$ 16[Suppl 3]:234-237 DOI 10.1007/s10039-013-2049-z

Online publiziert: 23. Januar 2014

(c) Springer-Verlag Berlin Heidelberg 2014

\title{
O. Ernst
}

Deutsche Gesetzliche Unfallversicherung, Landesverband Südwest, Heidelberg

Neuausrichtung des stationären Heilverfahrens der DGUV

\section{Umsetzung im Landesverband Südwest}

\section{Neues Schwerstverletzungs- artenverfahren (SAV)}

\section{Prüfverfahren}

Jedem Krankenhaus, das beim LV einen Antrag auf Beteiligung am SAV stellt, wird ein Antragsvordruck zugesandt. Mit dem Antrag sind u. a. besondere Qualifikationsnachweise der Ärzte in Hinblick auf ihre traumatologischen Kompetenzen vorzulegen. Ebenso sind ergänzende Informationen - z. B. die Pläne der Ambulanz/Schockräume und der Operationsabteilung - beizufügen.

An der Besichtigung vor Ort nimmt immer ein beratender Arzt des LV teil. Der Ablauf der Begehung richtet sich nach der Wegeführung eines Schwerstunfallverletzten. Beginnend vom Hubschrauberlandeplatz bzw. der überdachten Krankenwagenanfahrt führt der Weg insbesondere über die Notaufnahme (Schockräume, Computertomographie) in die Operationsabteilung und die Intensivstation. Neben der sächlichen Ausstattung werden auch die Prozesse beurteilt, insbesondere das Schockraummanagement. Beim Abschlussgespräch klärt der LV mit dem D-Arzt und dem Klinikträger das weitere Prozedere.

\section{Derzeitiger Stand - laufende Maßnahmen}

Bisher kündigten 16 Krankenhäuser beim LV einen SAV-Antrag an, und von
15 Krankenhäusern liegen Anträge vor (Stand 09.11.2013). Nach dem derzeitigen Stand der Prüfungen werden zum 01.01.2014 voraussichtlich 9 SAV-Beteiligungen in Baden-Württemberg und 2 SAV-Beteiligungen im Saarland ausgesprochen. Dabei handelt es sich um die im Folgenden aufgeführten Krankenhäuser und Kliniken (Stand 09.11.2013), wobei anzumerken ist, dass alle diese Einrichtungen als überregionale Traumazentren am TraumaNetzwerk ${ }^{\circledR}$ DGU (DGU: Deutsche Gesellschaft für Unfallchirurgie e. V.) beteiligt sind.

- Baden-Württemberg:

- Universitätsklinikum Freiburg,

- Städtisches Klinikum Karlsruhe,

- Klinikum Ludwigsburg,

- Krankenhaus St. Elisabeth Ravensburg,

- Katharinenhospital Stuttgart,

- BG-Unfallklinik Tübingen,

- Bundeswehrkrankenhaus Ulm,

- Universitätsklinikum Ulm,

- Schwarzwald-Baar-Klinikum, Villingen-Schwenningen,

- Saarland:

- Universitätskliniken des Saarlandes, Homburg/Saar,

- Klinikum Saarbrücken.

Diese voraussichtlichen SAV-Standorte sind auf der in $\square$ Abb. 1 dargestellten Karte als rote bzw. grüne Punkte dargestellt. Der grüne Punkt in der Mitte von Baden-Württemberg markiert die BGUnfallklinik Tübingen. Der weitere grüne 


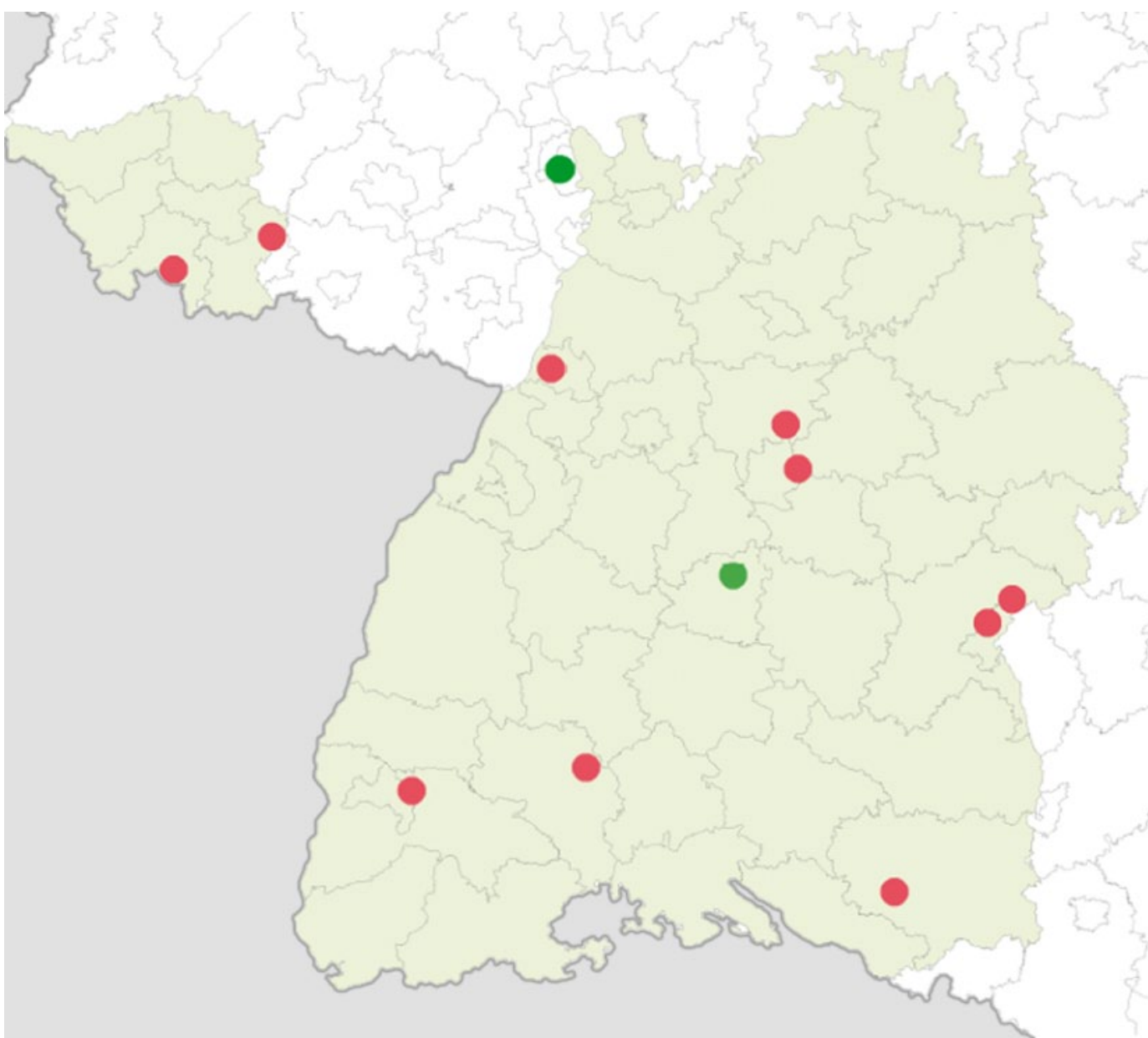

Abb. $1 \varangle$ Zulassung zum Schwerstverletzungsartenverfahren (SAV) im Landesverband Südwest, Stand 09.11.2013, rot künftige SAV-Kliniken, grün berufsgenossenschaftliche Kliniken (Deutsche Gesetzliche Unfallversicherung e. V., Referat Statistik)
Punkt liegt zwar in Rheinland-Pfalz; weil es sich hier um die BG-Unfallklinik Ludwigshafen handelt, die direkt an Nordbaden grenzt, ist diese mit aufgenommen.

Im Dezember 2013 werden die Landesverbände der DGUV alle am D-Arztund $\mathrm{H}$-Arzt-Verfahren ( $\mathrm{H}$ : an der besonderen Heilbehandlung beteiligt) Beteiligten in einem Rundschreiben ausführlich über die neuen Zuweisungsmechanismen informieren und die bundesweit am SAV-Verfahren beteiligten Krankenhäuser bekannt geben. Die EAP- (erweiterte ambulante Physiotherapie) und BGSWEinrichtungen (BGSW: berufsgenossenschaftliche stationäre Weiterbehandlung) werden parallel informiert, und die DGUV in Berlin wird die Unfallversicherungsträger ebenfalls über den Stand der Umsetzung in den Landesverbänden unterrichten.

Die strukturellen Veränderungen machen auch Anpassungen im Berichtswesen erforderlich. Zum 01.01.2014 werden deshalb die für das Heilverfahren erfor- derlichen Berichte, z. B. der D-Arzt-Bericht (F 1000), hinsichtlich des neuen SAV-Verfahrens geändert werden.

\section{Neues Verletzungsarten- verfahren (VAV)}

\section{Prüfverfahren}

Im LV sind derzeit 45 Krankenhäuser mit einem unbefristeten Vertrag am alten VAV beteiligt. Diese Häuser genießen eine Übergangsfrist und werden zu deren Ablauf am 31.12.2017 hinsichtlich der neuen VAV-Anforderungen geprüft. Bei einem Chefarztwechsel vor Ende der Übergangsfrist erfolgt die Prüfung zum Zeitpunkt dieses Wechsels.

Es gibt aber auch 29 Krankenhäuser, die derzeit befristet am alten Verletzungsartenverfahren beteiligt sind. Diese werden zum Ende ihrer vertraglichen Befristung - also bereits vor dem 31.12.2017 - geprüft.
Der Ablauf der Prüfung gestaltet sich folgendermaßen: Sobald der Prüfungstermin ansteht, sendet das Krankenhaus dem LV den neuen Antragvordruck zu. Wenn die VAV-Fallzahlen im Durchschnitt der letzten 5 Kalenderjahre unter 75 Fällen lagen bzw. in den letzten 3 Kalenderjahren nicht mindestens 75 Fälle erreichten [3], bittet der LV das Krankenhaus um eine interne Prüfung der VAV-Fallzahlen, denn deren Erreichen ist für die Beteiligung am neuen Verletzungsartenverfahren Bedingung. Weiterhin bittet der LV den Antragsteller um eine Stellungnahme, wenn ein Bewerber für das neue VAV-Verfahren nicht als regionales Traumazentrum im TraumaNetzwerk ${ }^{\circledast}$ DGU beteiligt sein sollte. Eine VAV-Beteiligung setzt eine solche Beteiligung als regionales Traumazentrum zwar nicht voraus, da sich die neuen VAV-Anforderungen allerdings sehr stark an den Anforderungen für ein regionales Traumazentrum anlehnen, interessieren den LV die Gründe, wenn der Antragsteller im TraumaNetzwerk ${ }^{\circledast}$ DGU 
nicht eine vergleichbare Versorgungsstellung einnimmt.

Werden die Voraussetzungen positiv beurteilt, erfolgt auch hier eine Begehung mit dem beratenden Arzt des LV. Deren Ablauf gleicht derjenigen im SAV-Verfahren. Sollte keine Beteiligung am neuen Verletzungsartenverfahren ausgesprochen werden können, prüft der LV, ob beim Wegfall dieses Hauses eine mögliche regionale Unterversorgung eintreten könnte. Ansonsten unterbreitet der LV das Angebot für die Beteiligung am stationären Durchgangsarztverfahren.

\section{Derzeitiger Stand}

Von Januar bis November 2013 wurden 7 Krankenhäuser nicht mehr vom alten in das neue Verletzungsartenverfahren übernommen. Alle wiesen eine Fallzahl von unter 75 Fällen aus, davon 6 eine Fallzahl von unter 50 Fällen. Die geringen Fallzahlen waren jedoch nicht der alleinige Grund für die Beendigungen der VAV-Beteiligungen. Beispielsweise waren die geforderten weiteren Unfallchirurgen nicht vorhanden oder es gab keine Hubschrauberlandemöglichkeit mehr.

\section{Neues stationäres Durchgangsarztverfahren (DAV)}

Alle am 31.12.2012 durchgangsärztlich besetzen, aber nicht am Verletzungsartenverfahren beteiligten Krankenhäuser dürfen bis zum Ende der Übergangsfrist am 31.12.2017 Unfallverletzte stationär behandeln. Fälle des neuen Verletzungsartenverzeichnisses sind weiterhin ausgenommen und müssen verlegt werden. Die Prüfung erfolgt bei einem Wechsel des D-Arztes bereits zu einem früheren Zeitpunkt.

Der Ablauf des Prüfverfahrens gleicht dem Grunde nach den bereits genannten Prüfverfahren (mit Ausnahme der Prüfung der Fallzahlen). Soweit keine weitere Beteiligung am neuen stationären DAV möglich ist, wird auch hier vom LV eine mögliche regionale Unterversorgung geprüft. Ansonsten wäre der D-Arzt - auch wenn er am Krankenhaus tätig ist - nur noch am ambulanten D-Arzt-Verfahren beteiligt.

Trauma Berufskrankh 2014 · 16[Suppl 3]:234-237 DOI 10.1007/s10039-013-2049-z

(c) Springer-Verlag Berlin Heidelberg 2014

\section{O. Ernst}

\section{Neuausrichtung des stationären Heilverfahrens der DGUV. Umsetzung im Landesverband Südwest}

\section{Zusammenfassung}

Hintergrund. Im Zuständigkeitsbereich des LV (Landesverband Südwest) der DGUV (Deutsche Gesetzliche Unfallversicherung e. V.) gibt es derzeit insgesamt 149 Krankenhäuser und Kliniken, die berechtigt sind, eine stationäre Behandlung von Arbeitsunfallverletzten durchzuführen. Spätestens zum 31.12.2017 ist von Seiten des LV vorgesehen, dass alle diese 149 Krankenhäuser hinsichtlich ihrer Beteiligungen an den neuen stationären Heilverfahren der DGUV geprüft sind. Zulassung zu den neuen Verfahren. Mit dem Antrag auf Zulassung zum Schwerstverletzungsartenverfahren (SAV) sind entsprechende Nachweise zur Qualifikation und Ausstattung vorzulegen. Für eine erneute Zulassung zum Verletzungsartenverfahren (VAV) sind ausreichend hohe VAV-Fallzahlen nachzuweisen. Der Ablauf der Begehung richtet sich bei beiden Prüfungen nach der Wegeführung eines Schwerstunfallverletzten, dabei werden die sächliche Ausstattung sowie die Prozessabläufe beurteilt. Die strukturellen Veränderungen machen auch Anpassungen im Berichtswesen erforderlich, dieses wird zum 01.01.2014 entsprechend angepasst. Krankenhäuser mit einem unbefristeten Vertrag am sog. alten Verletzungsartenverfahren oder die am 31.12.2012 durchgangsärztlich besetzt waren, behalten im Rahmen einer Übergangsfrist bis 31.12.2017 ihre bisherige Zulassung.

\section{Schlüsselwörter}

Unfallversicherung · Arbeitsunfall ·

Schwerstverletzungsartenverfahren

(SAV) - Verletzungsartenverfahren (VAV) .

Durchgangsarztverfahren (DAV)

\section{New structure of the inpatient medical treatment of the German statutory accident insurance (DGUV). Implementation in the Southwest Regional Association}

\begin{abstract}
Background. In the catchment area of the Southwest Regional Association (LV) of the German statutory accident insurance (DGUV) there are currently a total of 149 hospitals and clinics which are qualified to carry out inpatient treatment of patients with occupational accident injuries. Before 31 December 2017 at the latest the LV has the intention of auditing all 149 of these hospitals with respect to participation in the new inpatient medical treatment procedure of the DGUV. Accreditation for the new procedures. Applications for accreditation for the severely injured type procedures (SAV) must be accompanied by the necessary documentation on qualifications and instrumentation. For renewed approval for the injury type procedure (VAV) the necessary VAV case numbers must be fulfilled. In both procedures the inspec-
\end{abstract}

tion is oriented to the route taken by severely injured trauma patients whereby the equipment, facilities and process operation will be assessed. The structural alterations also necessitate adjustment in the documentation process which will be correspondingly adapted before 1 January 2014. Hospitals with an unlimited contract in the so-called old VAV or those which employed accident insurance consultants on 31 December 2012, will retain the previous approval over a transition period until 31 December 2017.

\section{Keywords}

Accidents, occupational - Accident insurance · Severe injury type procedure (SAV) - Injury type procedure (VAV) · Accident insurance consultant procedure (DAV)

\section{Probleme bei der praktischen Umsetzung/Auffälligkeiten}

Der LV stellte bei der Umsetzung der neuen stationären Heilverfahren Probleme fest, von denen im Folgenden einige erläutert werden:
Fallzahlen. Der Nachweis der VAV-Fallzahlen über die D-Arzt-Statistik, die über DALE-UV (Datenaustausch mit Leistungserbringern in der gesetzlichen Unfallversicherung) ausgewertet wird, bereitet Probleme. Bei den dem LV vorliegenden Fallzahlen handelt es sich um die Zahlen der über den elektronischen 
Datenaustausch übermittelten D-ArztBerichte. Diese können im Einzelfall von den tatsächlichen Fallzahlen abweichen: Wenn beispielsweise ein VAV-Fall im D-Arzt-Bericht nicht als solcher verschlüsselt wurde, wird dieser auch nicht über DALE-UV gezählt. Der LV gibt deshalb jedem Krankenhaus die Möglichkeit, die vorliegenden Fallzahlen intern zu überprüfen.

Räumliche Ausstattung. In vielen älteren VAV-Krankenhäusern befindet sich der Computertomograph nicht in unmittelbarer Nähe zum Schockraum. Hier arbeitet der LV mit Auflagen, wonach dies bei den nächsten Umbaumaßnahmen in der Notaufnahme behoben werden muss.

Hubschrauberlandeplatz. Bei der Prüfung der Antragsteller im neuen SAVVerfahren fiel auf, dass sich bei einigen Krankenhäusern der Hubschrauberlandeplatz (im 24-h-Betrieb) nicht in räumlicher Nähe zu den Schockräumen befindet. Der LV begrüßt es sehr, dass bei allen betroffenen Kliniken bereits konkrete Planungen vorliegen oder bauliche Maßnahmen begonnen wurden, um durch einen neuen Hubschrauberlandeplatz auf dem Dach die Transportzeiten für die Verletzten zum Schockraum zu minimieren. Die jeweiligen SAV-Verträge werden mit einer entsprechenden Auflage versehen.

Fachärzte. Im neuen Verletzungsartenverfahren werden neben dem Durchgangsarzt 2 weitere Unfallchirurgen gefordert [2] -statt wie bisher ein weiterer Unfallchirurg. Hier deutet sich an, dass insbesondere kleinere Kliniken Probleme haben, diesen personellen Mehrbedarf nachzuweisen.

Während der Begehungen durch den LV wurden wiederholt sog. Auffälligkeiten festgestellt:

Nicht abgesprochene bauliche Veränderungen. In den letzten Jahren wurden in manchen Krankenhäusern bauliche Veränderungen vorgenommen, ohne dass der LV hiervon unterrichtet oder bei den Planungen mit einbezogen worden war. Änderungen - insbesondere baulicher Art sind dem LV jedoch unverzüglich zu melden. Es empfiehlt sich, den jeweils zustän- digen Landesverband bereits in der Planungsphase mit einzubinden.

Suboptimale Bauplanung. Teilweise wurden bei aktuellen Neu- oder Umbauten die Wegeführung und die Wegstrecken für den Transport des Schwerstunfallverletzten suboptimal geplant und umgesetzt, insbesondere, wenn beim Schockraum und dem Raum für den Computertomographen keine Wand-an-Wand-Lösung verfolgt wurde.

Hygiene. Bei den Begehungen in den Operationsabteilungen zeigte sich, dass eine deutliche Diskrepanz zwischen den im Hygieneplan niedergelegten Verhaltensregeln und der gelebten Realität bestehen kann. In diesen Fällen wurden u. a. die betroffenen Durchgangsärzte daran erinnert, auf diese Problematik besonders zu achten.

\section{Mangelnde Unterstützung durch Kran-} kenhausleitung. Schließlich fiel dem LV bei den bisherigen Prüfungen auf, dass es immer wieder kaufmännische Krankenhausleitungen gibt, die sich der Bedeutung als Vertragspartner der DGUV nicht ganz bewusst sind. Dies zeigt sich beispielsweise, wenn bei den Gesprächen und Besichtigungen kein Vertreter der kaufmännischen Krankenhausleitung teilnimmt oder wenn der D-Arzt von seiner Verwaltung nicht in dem Maße unterstützt wird, wie es für eine optimale akuttraumatologische Versorgung Arbeitsunfallverletzter eigentlich erforderlich wäre.

\section{Korrespondenzadresse}

\section{Ernst}

Deutsche Gesetzliche Unfallversicherung, Landesverband Südwest, Kurfürsten-Anlage 62, 69115 Heidelberg Olaf.Ernst@dguv.de

\section{Einhaltung ethischer Richtlinien}

Interessenkonflikt. O. Ernst gibt an, dass kein Interessenkonflikt besteht.

Dieser Beitrag beinhaltet keine Studien an Menschen oderTieren.

The supplement containing this article is not sponsored by industry.

\section{Literatur}

1. Deutsche Gesetzliche Unfallversicherung (2013) DGUV Statistik 2012. DGUV, Berlin

2. Deutsche Gesetzliche Unfallversicherung (2013) VAV-Anforderungen in der Fassung vom 01.01.2013, Nr. 2.2.1. DGUV, Berlin

3. Deutsche Gesetzliche Unfallversicherung (2013) VAV-Anforderungen in der Fassung vom 01.01.2013, Nr. 4.4.4. DGUV, Berlin 